to be published in Nucl. Instr. and Meth. B

\#107

\title{
In-air PIXE analysis by means of glass capillary optics
}

\author{
Takuya NEBIKI, M Hasnat KABIR and Tadashi NARUSAWA \\ Kochi University of Technology \\ Tosa-Yamada, Kochi 782-8502 JAPAN
}

\begin{abstract}
A novel technique to introduce high energy ion beams to atmospheric environment is presented, which enables in-air PIXE measurements. Slightly tapered glass capillary optics is applied to work as a differential pumping orifice as well as a focusing lens. The flux intensity is enhanced by at least one order of magnitude due to the focusing effect. Using capillaries of 10-20 microns outlet diameters, we obtain several hundreds $\mathrm{pA}$ of $4 \mathrm{MeV}-\mathrm{He}^{++}$ion beam and apply it to PIXE analysis of the seabed sludge without any sample treatments. A comparison of spectra between wet and dry sludge samples suggests the usefulness of our new technique.
\end{abstract}

PACS: 34.50.Dy, 61.18.Bn, 68.49.Sf, 82.80.Yc

Key Words: PIXE, Glass capillary optics, Environmental pollutions, In-air measurements Postal coke: Tosa-yamada, Kochi 782-8502, Japan

e-mail address: narusawa.tadashi@kochi-tech.ac.jp 
to be published in Nucl. Instr. and Meth. B

FAX: $+81-887-57-2120$ 
to be published in Nucl. Instr. and Meth. B

\section{Introduction}

Particle Induced X-ray Emission Spectrometry (PIXE) is a well-known analytical method and its application ranges a variety of fields[1]. The extremely high multi-elemental sensitivity of PIXE measurements makes it ideal for e.g. an environmental pollution monitor. However, since the measurements usually must be carried out in vacuum, we encounter difficulties in many cases. For instance, wet samples or liquid samples are difficult because they are not compatible with vacuum, and complex procedures of sample preparation are often needed. In-air PIXE attracts increasing interests because we can collect PIXE data without such procedures. In order to carry in-air PIXE measurements many attempts have been done [2-4]. The most popular is to use a very thin polymer or metal foil as the boundary of vacuum and air. High energy ion beams penetrate the foil and hit the sample in the air having enough beam energy and flux intensity for analyses. Once the foil breaks, however, causing big damage to the accelerator. The risk is often avoided by using extra parts such as fast valves.

The glass capillary optics is an interesting and useful lens for ion beams. Fabrication procedures of the optic and the fundamental ion transmission characteristics have been reported elsewhere[5]. High energy ion beams of $\mathrm{MeV}$ range are effectively focused by the capillary, and most interestingly the energy loss of ions in the course of transmission is negligibly small. The capillary was originally designed as an artificial channel for ions, but 
to be published in Nucl. Instr. and Meth. B

the results of our preliminary experiments briefly described above indicate that the mechanism of ion deflection at the wall surface is not merely by the nuclear-nuclear interaction but probably also by the electric charge effect [6].

In the present paper, we describe a new attempt to utilize the glass capillary optics for in-air PIXE analysis of wet samples such as the seabed sludge. In this application the capillary works as a focusing lens of the ion beam as well as an orifice for differential pumping. We show that the ion beam is successfully introduced to the atmospheric environment, and in-air PIXE measurements can be carried out without any difficulties, although more optimization of the experimental conditions is needed.

\section{Results and discussion}

The glass capillaries used in the present study have an inlet diameter of $0.8 \mathrm{~mm}$ and an outlet diameter of 10-20 microns, and a length of a few $\mathrm{cm}$. The taper angle is therefore about 1 degree. The glass capillary molded into an aluminum pipe is mounted on a 4-axis goniometer. The goniometer adjusts $\mathrm{x}, \mathrm{y}$ positions and the tilt angle of the capillary with respect to the ion beam line. The conductance of the capillary is roughly estimated to be in the order of $10^{-6} 1 / \mathrm{s}$ when the outlet diameter is 10 microns, which is small enough provided that we use a 100 1/s turbo-molecular pump as the differential pump. Typical $\mathrm{He}^{++}$ion beam current before and after the capillary is $10 \mathrm{nA}$ and $200 \mathrm{pA}$, respectively. This corresponds to 
to be published in Nucl. Instr. and Meth. B

an enhancement of the beam flux intensity by $\sim 2$ orders. The ion beam current at the sample stage was a function of the capillary-sample stage distance, in accordance with the literature values of ion range in air [7]. Figure 1 shows the actual arrangement of the glass capillary (A), x-ray detector (B), and the sample: a seabed sludge droplet (C). The top of the capillary is barely seen but it is only $1 \mathrm{~mm}$ or less apart from the sample surface. The distance between the detector and the sample is also set as close as possible but it is limited to $\sim 2 \mathrm{~cm}$ by the detector housing diameter. Though proton beams of 2-3 $\mathrm{MeV}$ are usually used for PIXE analysis, we used a $\mathrm{He}$ ion beam in the present study because of some regulations in our laboratory.

In order to check the feasibility of in-air PIXE analysis, we first measured a $\mathrm{Ga}_{0.7} \mathrm{In}_{0.3} \mathrm{~N}_{0.01} \mathrm{As}_{0.99}$ quaternary crystal sample mounted on a $\sim 20$ microns thick carbon foil. Resulting spectra are shown in Fig.2 for incidence energies of 2 and $4 \mathrm{MeV}$. We notice that the $4 \mathrm{MeV}$ spectrum (Fig.2 (a)) is much better than the $2 \mathrm{MeV}$ spectrum (Fig.2 (b)) because of the ionization cross section difference. In the $4 \mathrm{MeV}$ spectrum we see 3 peaks that cannot be assigned to GaInNAs. Peak (A) corresponds to Si, and this is most likely from the glass capillary itself. Since the glass wall thickness near the top of capillary is around 10 microns, Si x-rays emitted inside the capillary have some possibilities to penetrate the wall towards the detector, also there is a small probability that x-rays generated inside the capillary come out through the outlet and reflect at the sample surface, or scattered ions can hit the outside 
to be published in Nucl. Instr. and Meth. B

of the capillary. These ions can excite Si x-rays. Peak (B) looks like a shoulder but its energy corresponds to Ar. This may be due to Ar gas in the air. Peak (C) is clearly from Fe. We suppose that this is again due to scattered ions or reflected x-rays. In spite of these possible drawbacks, the present technique has a great merit that external microbeams are easily obtained.

Uranouchi Bay of Kochi prefecture, Japan is a scenic resort and also well-known for fish-farming. People grow various fish like sea bream and young yellowtails. In spite of the beautiful scenery, the seabed of this bay is not very clean. In Fig.3 a PIXE spectrum of the seabed sludge (b) is compared with a reference spectrum obtained from the sea water (a). Measurements were done in vacuum. As clearly shown in the spectrum, the seabed sludge contains several heavy metal elements such as $\mathrm{Fe}, \mathrm{Ti}, \mathrm{V}, \mathrm{Mn}, \mathrm{Cu}$, and $\mathrm{Zn}$, which are not seen in the sea water spectrum. We are not sure about the source of these elements, but it is likely that these are due to the long time accumulation of the leftover of fish-foods.

Figure 4 compares PIXE spectra from dried (a) and liquid (b) seabed sludge, both measured in the air. We see overall agreements in two spectra, but relative intensities differ considerably from each other. Particularly the Cl-K x-ray intensity in (a) is larger than in (b) by one order of magnitude. The origin of this difference is not clear, but one possibility would be that most elements are condensed to a superficial layer in the course of drying, thus 
to be published in Nucl. Instr. and Meth. B

giving relatively high counting rates in (a). In contrast with this, most elements are distributed diluted in the water in (b), and the incoming ions lose their energy until they reach the target atoms, thus resulting in a lower x-ray generation cross sections. It is interesting to note that the Si-K x-ray intensity is alike in both cases. This suggests that this Si signal results from some artifacts as mentioned earlier. In any case, comparing Fig. 4 with Fig.3, we admit that the quality of in-air measured PIXE spectra is far inferior to those measured in vacuum. In order to obtain useful quantitative results by in-air measurements, we need to optimize the experimental conditions, such as ion species, the ion beam energy, the way to hold the samples, the environment around the sample, etc.

\section{Conclusion}

We have proposed a unique and simple way to obtain PIXE spectra from virtually any type of samples: solids, liquids and gases, and demonstrated the feasibility by showing results of seabed sludge measurements. The present facility is certainly useful for PIXE analysis of various samples that are not compatible with the vacuum environment. However, some improvements are clearly needed for practical use of the facility; the use of a $2 \sim 3 \mathrm{MeV}$ proton beam instead of a $4 \mathrm{MeV} \mathrm{He}^{++}$beam enhances the detection sensitivity and ion beam ranges in the air, the environment around the sample should be flowing He gas in order to enhance the ranges of ions and x-rays, as well as to avoid dust particles or water vapor interfering with the measurements. 
to be published in Nucl. Instr. and Meth. B 
to be published in Nucl. Instr. and Meth. B

\section{References}

[1] S..A.E.Johansson, J.L.Campbell, K.G.Malmqvist, Ed., Particle-Induced X-ray Emission Spectrometry (PIXE)”, Wiley \&Sons, New York, 1995.

[2] J. Räisänen, Nucl. Instr. and Meth. 231 (1984) 220.

[3] Ruvalcaba Sil J.L., Monroy M., Morales J.G. and López K., IX International Conference on PIXE, Guelph, Canada, 2001.

[4] Vijayan, V., Choudhury, R. K., Mallick, B., Sahu, S., Choudhury, S. K., Lenka, H. P., Rautray, T. R., Nayak, P. K., Current science, 85(6) (2003) 772.

[5] T,Nebiki, T.Yamamoto, T.Narusawa, M.B.H.Breese, E.J.Teo, F.Watt, J. Vac. Sci. Technol. A21 (2003) 1671.

[6] N.Stolterfoht, J.-H.Bremer, V.Hoffmann, R.Hellhammer, D.Fink, A.Petrov, and B.Sulik, Phys. Rev. Lett. 88 (2002) 133201-1

[7] J. F. Ziegler, Range of Ions in Matter, <http://www.srim.org/SRIM/Ranges.htm> 
to be published in Nucl. Instr. and Meth. B

Figures caption

Fig. 1. Photograph of (A) the glass capillary optics, (B) the X-ray detector and (C) the liquid sludge droplet.

Fig. 2. In-air PIXE spectra of the GaInNAs sample using (a) a $4 \mathrm{MeV} \mathrm{He}^{++}$and (b) a $2 \mathrm{MeV}$ $\mathrm{He}^{+}$beam. The ion beam dose is $3 \mu \mathrm{C}$.

Fig. 3. PIXE spectra of (a) sea water and (b) seabed sludge. These spectra are measured in vacuum after complicated preparation procedures of the samples. The ion beam is 4 $\mathrm{MeV}-\mathrm{He}^{++}$and the dose is $1.4 \mu \mathrm{C}$.

Fig. 4. Comparison of in-air PIXE spectra. (a) dried sludge and (b) liquid sludge sample. 
to be published in Nucl. Instr. and Meth. B

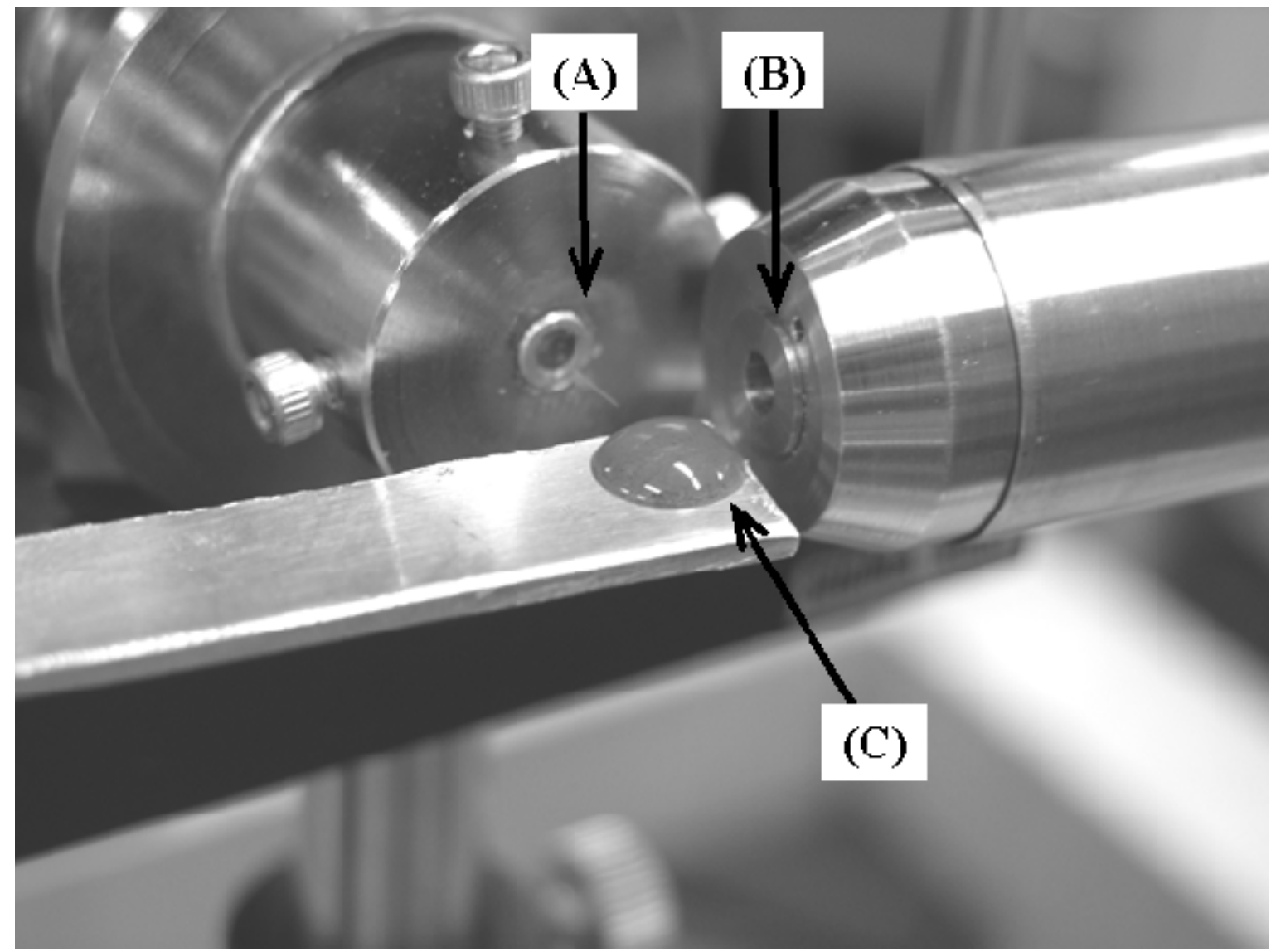

Figure 1 
to be published in Nucl. Instr. and Meth. B
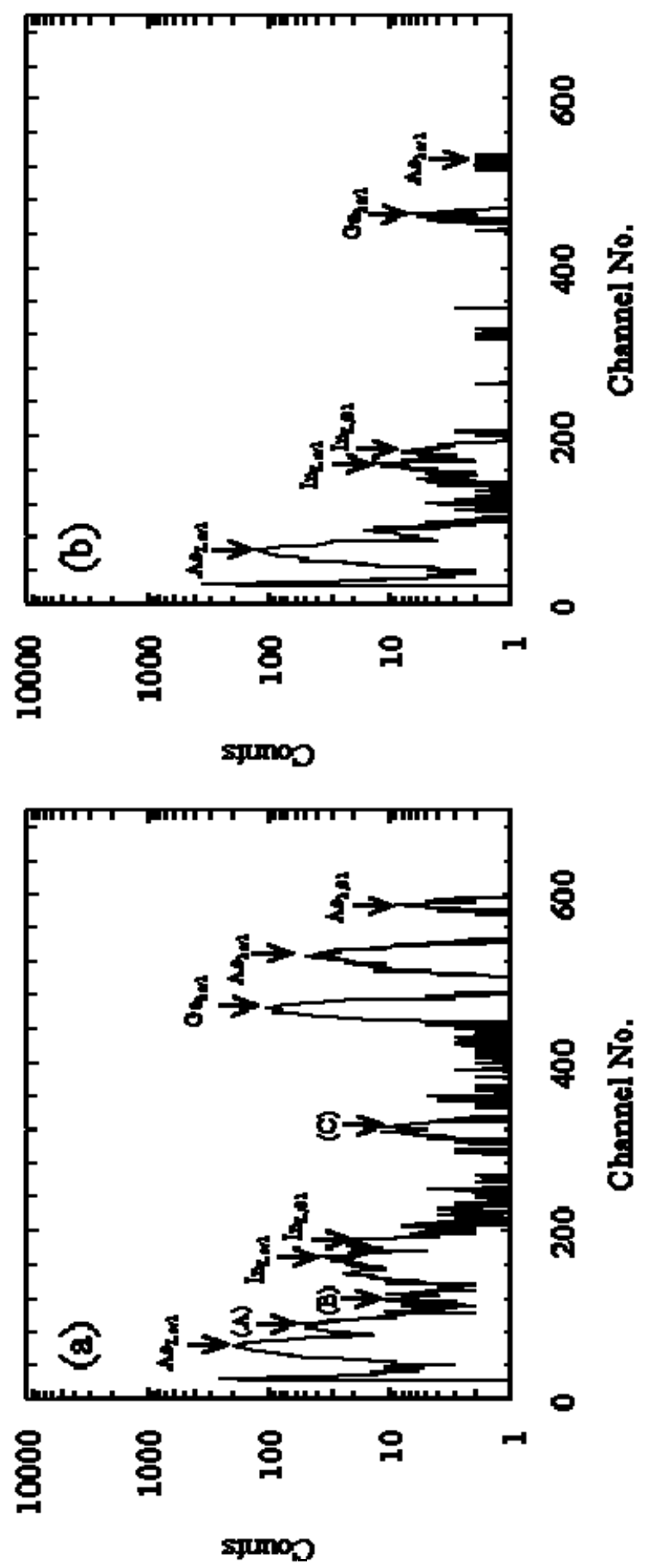

Figure 2 
to be published in Nucl. Instr. and Meth. B
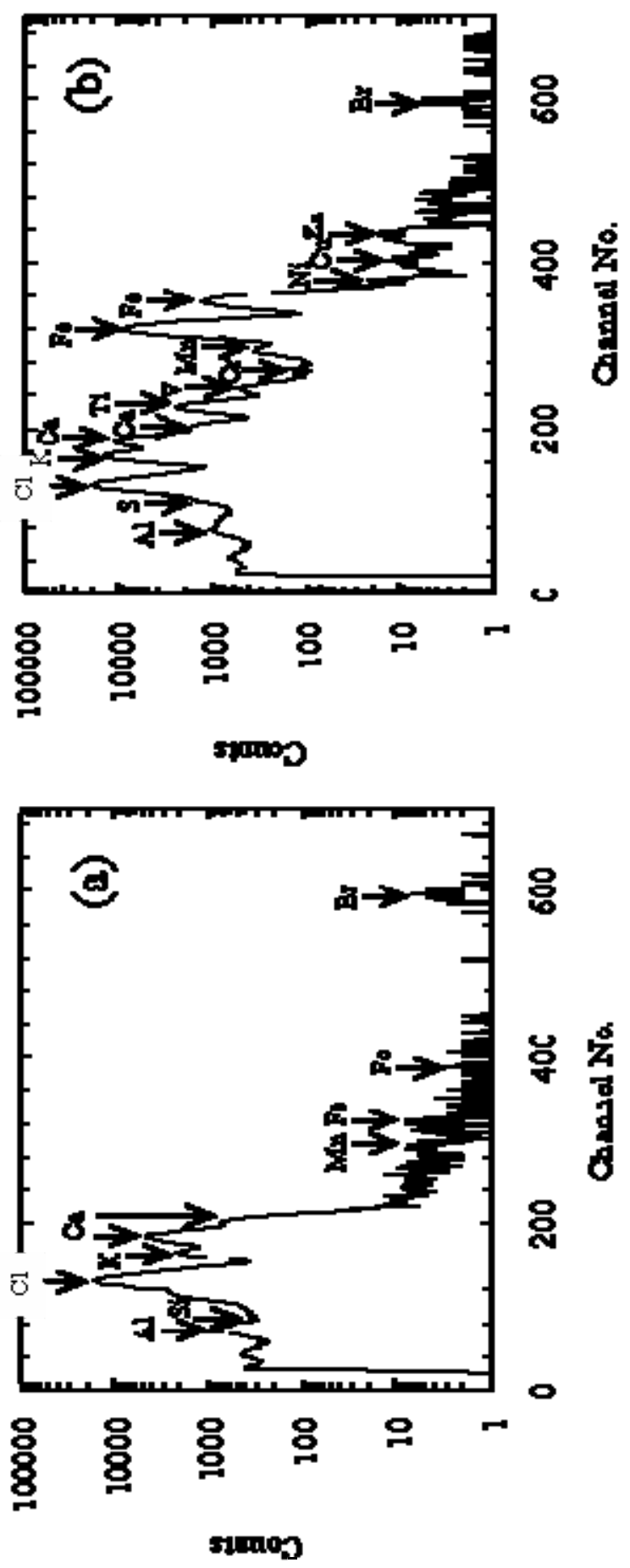

Figure 3 

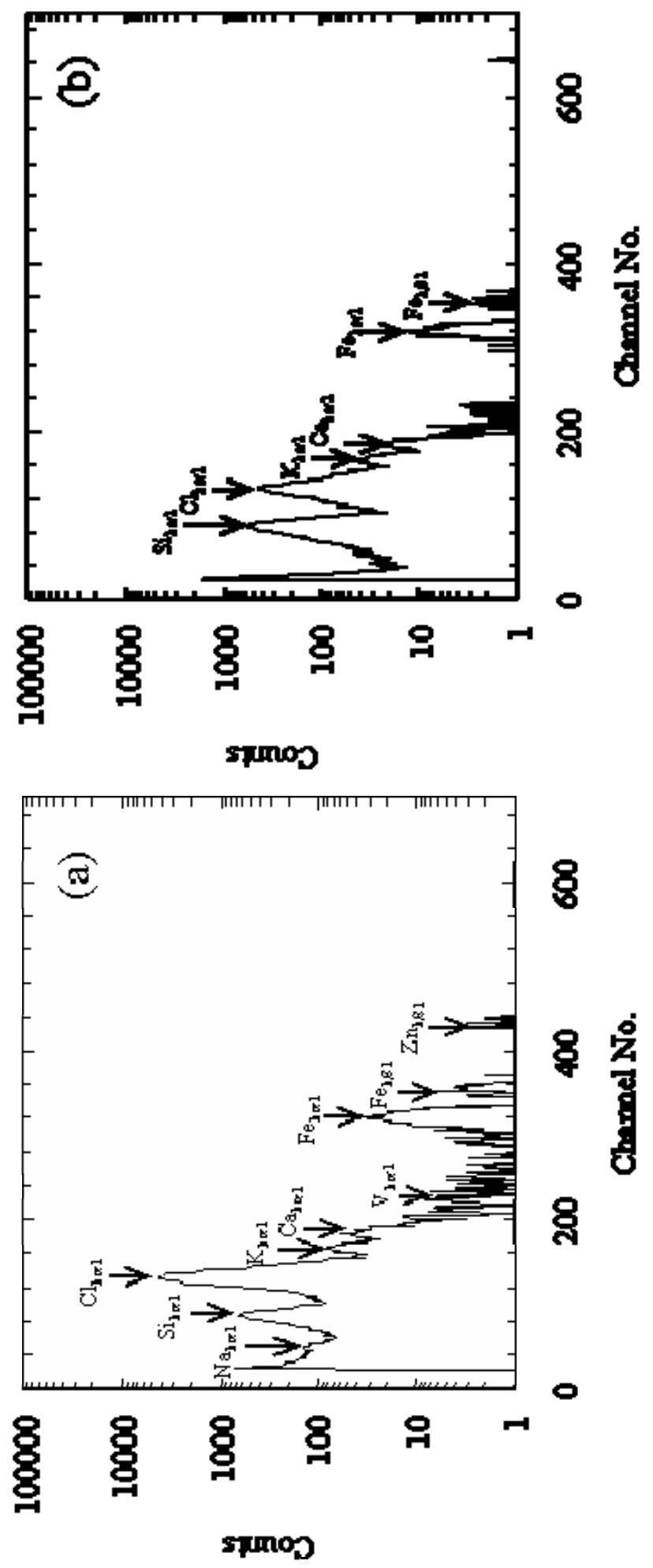

Figure 4 